Blanco, M.P. y Garrido, M.C. (2013). Lenguas de especialidad e interacción oral en el sector turístico: Reflexiones sobre su implicación en el Espacio Europeo de Educación Superior. Revista Electrónica Interuniversitaria de Formación del Profesorado, 16(1), 25-40.

DOI: http://dx.doi.org/10.6018/reifop.16.1.179421

\title{
Lenguas de especialidad e interacción oral en el sector turístico: Reflexiones sobre su implicación en el espacio europeo de educación superior
}

\author{
María del Pilar Blanco Calvo, María del Carmen Garrido Hornos \\ Universidad de Valladolid
}

\section{Resumen}

El proceso de enseñanza-aprendizaje de lenguas extranjeras en los estudios universitarios de Grado en Turismo ha sufrido cambios significativos. En este artículo se analizan sus consecuencias, tanto en la búsqueda de modelos pedagógicos en consonancia con el Espacio Europeo de Educación Superior - que promuevan la autonomía en el aprendizaje-, como en la adaptación de las lenguas de especialidad a las necesidades lingüísticas, pragmáticas, sociolingüísticas y funcionales características del sector profesional turístico.

Esta acomodación a las necesidades de los discentes, futuros profesionales en el sector turístico, implica detenerse en la importancia del desarrollo de la competencia comunicativa oral, pero también en las dificultades psicolingüísticas que contempla dicho aprendizaje.

\section{Palabras clave}

Enseñanza superior; competencias; lenguas de especialidad; interacción comunicativa; turismo.

\section{Languages of specialization and oral interaction in the tourism sector: Reflections upon its implication in the higher education area}

\section{Abstract}

The second languages teaching and learning process in the university studies of Degree in Tourism has suffered important changes. In this paper we analyse their consequences, both in the search of 
pedagogical models in line with the European Higher Education Area — changes which foster autonomous learning - and in the adaptation of the languages of specialization to the linguistic, pragmatic, sociolinguistic and functional necessities typical of the tourism sector.

This adjustment to the learners' needs — prospective professionals of the tourism field- implies a reflection upon the transcendence of the development of the oral communicative competence, but also upon the psycholinguistic difficulties involved in this type of learning.

\section{Key words}

Higher Education; competences; languages of specialization; communicative interaction; tourism.

De la libertad de explorar nace la alegría de aprender. Del conocimiento adquirido mediante la iniciativa personal nace el deseo de saber más cosas. (E.O. Wilson, en Rué, 2009: 47)

\section{Introducción}

Inmersos como estamos en las nuevas tendencias de la enseñanza universitaria, el aprendizaje de lenguas extranjeras por medio de competencias ha dejado de ser un utópico sistema para convertirse en una metodología realista. Hemos emprendido un proceso de reajuste, renovación y adecuación a las estructuras de los estudios superiores de segundos idiomas, algo no realmente novedoso en nuestro ámbito de formación, pues ya desde los años 70 el campo de didáctica de las lenguas ha dispuesto de una ingente cantidad de estudios en los que los términos "comunicación" y/o "competencia comunicativa" figuran de forma sistemática".

Bernal (2006) considera que el desarrollo de las competencias universitarias en la enseñanza de lenguas - entendidas como objetivos precisos y directos - ha de potenciar el aprendizaje autónomo del alumno y capacitarlo en su prospectiva inserción al mercado laboral. La adquisición de las competencias, según este autor, está estrechamente vinculado a:

a) Un saber conceptual, con un correspondiente desarrollo de las capacidades profesionales.

b) Un saber procedimental, en relación a la plasmación empírica de los conocimientos adquiridos.

c) Un saber actitudinal, entroncado con la convivencia profesional y la mutua colaboración.

d) Un saber metacognitivo, vinculado, a su vez, a la capacidad de autocrítica, autonomía y responsabilidad académico-profesional.

Ya sea contemplando la valoración de calidad profesional de Bernal, ya asimilando la acepción académica de las competencias como una combinación de conocimientos, habilidades y valores de formación universitaria, resulta claro que el mundo del turismo —íntimamente ligado al estudio de idiomas por razones obvias - requiere del empleo fluido de la lengua meta como vehículo de comunicación y transmisión de conocimientos tanto académicos como profesionales. La finalidad del profesor de idiomas en este sentido no es otra que proporcionar las estrategias que faculten al discente para interactuar lingüísticamente en cualquier contexto social y cultural que afronten una vez concluidos sus estudios universitarios.

\footnotetext{
${ }^{1}$ Por señalar sólo a los pioneros, apuntaremos que ya en 1975, la obra The Threshold Level in a European Unit/Credit System for Modern Language Learning by Adults., de Van Ek se encumbró como la primera enunciación manifiesta de propósitos didácticos dependientes de la adecuación sociolingüística. Este tratado en inglés, que recoge una enumeración detallada de actos de habla que han de ser asimilados y empleados por el alumno como prueba de su competencia en la lengua que estudia, fue el precursor de otros tantos equivalentes en las distintas lenguas europeas: en 1976 Coaste escribe para la lengua francesa Le Niveau Seuil; Slagter hizo lo propio con Nivel Umbral para el español; Livello Soglia se proyectó como la guía para el italiano y Kontakt Schwelle resultó ser el equivalente para el alemán.
} 
Para la consecución de tales objetivos pedagógicos, hemos precisado de una nueva canalización en el modo de comprender la enseñanza de idiomas, pues aun cuando las metodologías activas y comunicativas nos han acompañado desde años atrás, la orientación y los nuevos modelos de tutoría universitaria han supuesto un replanteamiento de nuestros procedimientos didácticos, de nuestras planificaciones docentes; en definitiva, de nuestra forma de entender la universidad. Se aboga por unos estudios científicos más especializados en los que el profesor se transforma en, como sugeriremos, un "formador competente" tan preocupado por los saberes conceptuales como por los conocimientos profesionales - “saber hacer" será un concepto nuclear de nuestra aproximación.

El propósito de este estudio es reflexionar sobre la viabilidad y utilidad práctica de las competencias genéricas, instrumentales, personales y sistémicas de las materias de idiomas en las aulas de Grado de Turismo, debatiendo hasta qué punto puede ver el estudiante satisfechas sus habilidades comunicativas - ya sean éstas escritas u orales- dentro del panorama académico de reciente implante y dónde recaen las restricciones propias de un nuevo sistema educativo que, por si fuera poco, se está viendo gravemente afectado por la recesión económica que atraviesa nuestro país y los recortes derivados de ella.

En el marco de los Proyectos de Aprendizaje Tutorado, Metodologías Activas y Aprendizaje Colaborativo — tan centrados todos ellos en el "leitmotiv" "enseñar a aprender"—, ¿pueden suplirse de algún modo las limitaciones ocasionadas por la centralización en el trabajo autónomo del alumno? ¿Dónde acaban nuestras funciones como "transmisores de conocimientos” y dónde empieza nuestra responsabilidad como "desarrolladores de competencias”? ¿Realmente existe esa función? ¿Estamos respondiendo seriamente a las necesidades formativas de nuestros estudiantes en sus fines específicos?

En un panorama económico como el presente, que -en lo concerniente a los recortes a nivel contractual- no permite con frecuencia el fraccionamiento de líneas ni grupos y eleva la ratio al total de la matrícula, tanto profesor como alumno del Grado en Turismo son conscientes de la voluntad y el afán de superación que exige la adquisición de una lengua extranjera, si bien asumen la dificultad de lograr su objetivo y desarrollar las competencias demandadas. Así, la práctica oral —a la que se dedicará en nuestras páginas un epígrafe íntegro- se complica no sólo por factores psicolingüísticos propios del ensayo de la destreza, sino también por cuestiones prácticas como la imposibilidad de fomentar la fluidez de todos los estudiantes por falta material de tiempo.

En este artículo revisaremos éstas y otras condiciones, centrándonos, para ello, en las asignaturas de lenguas extranjeras del Grado en Turismo por ser equipo docente activo de la titulación ${ }^{2}$ y, sobre todo, por entender que los conocimientos de las lenguas no se circunscriben en este punto a un uso instrumental del idioma, sino a un empleo específico del vehículo de interacción lingüística. Atención expresa recibirán estas asignaturas por su calidad de lengua de especialidad y se detallarán, como veníamos adelantando, elementos propios del manejo de una lengua que no se limita al simple intercambio de conocimientos entre profesionales del sector; muy al contrario, deliberaremos acerca de los riesgos de este tipo de aprendizaje, que, con frecuencia tiende a obviar ciertas competencias para focalizar su atención en el léxico específico.

Para finalizar, analizaremos las dificultades propias del entrenamiento de la producción oral: las fuentes de ansiedad en el manejo de la destreza tratarán de dar respuesta a la cuestión de partida: ¿es posible que el alumno adquiera las competencias que, inicialmente, calculamos? Todas nuestras teorías y conclusiones tendrán como punto de partida las competencias desglosadas en el Libro Blanco del Grado en Turismo de ANECA (2004) —al que haremos referencia continua_, entendiendo éste como decálogo-guía para la construcción de las asignaturas de nuestra titulación.

\footnotetext{
2 El artículo que el lector va a comenzar es un planteamiento teórico, una reflexión escrita y basada en la experiencia en el aula de lenguas extranjeras del Grado en Turismo. Recogemos en estas páginas las conclusiones derivadas de prácticas reales y de las vivencias en el desarrollo de las materias de las que las autoras somos responsables.
} 


\section{Aprendizaje de lenguas extranjeras en el grado en turismo}

Los nuevos estudios de Grado en Turismo, partiendo de la realidad y del acercamiento al hecho turístico, buscan atender "la propia evolución del turismo, la demanda de sectores cada vez más expertos y las necesidades de los destinos de acogida" (ANECA, 2004, p. 25). Se demandan profesionales con una formación específica y diferenciada, con una visión amplia del sector turístico y de ocio de manera que "la formación en turismo debe ir adaptándose continuamente a los nuevos postulados turísticos" (ANECA, 2004, p. 25).

En la memoria del Programa Verifica de los estudios de Grado en Turismo de la Universidad de Valladolid (sin publicar) queda patente que, como profesores responsables, tratamos de ofertar "unos estudios científicos más especializados que tengan relevancia en el mercado laboral nacional y europeo, que fomenten la movilidad y la empleabilidad de los alumnos, proveedores de la máxima calidad". Éstos son los objetivos de la titulación y, dentro de sus ellos, "calidad" es el término clave.

Cano González (2009), compañero del Departamento de Pedagogía de nuestra universidad y estudioso de los nuevos modelos de tutoría y el aprendizaje por competencias, propone una reflexión de las nuevas corrientes en base a, por una parte, la reforma en sí —con la complejidad estructural de los nuevos planes de estudio (Grado, Máster y Doctorado)— y, por otra, al perfil propio del alumnado, señalando:

a) El aumento del número de estudiantes en las últimas décadas

b) La heterogeneidad de los alumnos en relación al nivel académico y personal

c) El creciente número de discentes que compatibilizan trabajo y estudio

d) El absentismo generalizado en las sesiones formativas

e) Y su subsiguiente fracaso académico y/o abandono de estudios (Cano González, 2009, p. 187).

Cano entiende que "las competencias se van adquiriendo por un proceso de aprendizaje continuo y dinámico (... ) unido a nuestra vida y actividad" (Cano González, 2009). Y, de hecho, la preocupación del cuerpo docente de idiomas por una enseñanza de calidad no sólo atiende a la problemática inicial de la precisión en el uso de la segunda lengua, ni siquiera a la cobertura y exposición exitosa de los diseños curriculares de las asignaturas de las que somos responsables. Académicos especialistas subrayan la relevancia que la calidad del trabajo autónomo del discente ha de tener en toda asignatura —especialmente en las pertenecientes al segundo ciclo de la titulación-, pues según el Espacio Europeo de Educación Superior, el profesor es, antes que ilustrador de conocimientos, formador vocacional de personas: su función primaria ha de ser enseñar a aprender, instruir para gestionar y ser formador competente.

El nuevo Plan de Estudios del Grado en Turismo señala ya desde sus primeras líneas en el Libro Blanco del Título de Grado en Turismo la relevancia que, por ejemplo, se le ha de conceder a la asignatura de Inglés, concebida ésta ya desde sus niveles iniciales como una inmersión bilingüe que capacite al estudiante en prospectivas comunicaciones escritas y/u orales en la lengua meta: "[I]a evidente implantación de la lengua inglesa — dicen sus líneas relacionadas con las competencias específicas relacionadas con el Inglés- en los diferentes ámbitos profesionales y muy especialmente en el entorno turístico, hace imprescindible su conocimiento y uso" (ANECA, 2004, p. 132). Se trataría, según vemos, de un "perfeccionamiento" y una "intensificación” del inglés que permita al alumno trabajar en medios socioculturales diferentes con una marcada orientación del servicio al cliente. Para ello, y como reiteraremos a lo largo del presente artículo, el manejo correcto de las técnicas de comunicación ha de trabajarse ya desde las primeras asignaturas del currículo pues, bien se recoge a continuación en lo que a los Conocimientos Profesionales se refiere ("saber hacer"), se esperará del alumno:

a) Comunicarse en inglés en cualquier medio. Saber comprender textos originales relacionados con temas turísticos

b) “Trabajar” en lengua inglesa en un entorno profesional 
C) Comprender y redactar documentos, informes y memorias en un lenguaje técnico turístico (ANECA, 2004).

En el caso de las segundas lenguas extranjeras, se persigue desarrollar la competencia oral y escrita adaptándolas a "situaciones profesionales cotidianas como instrumento de comunicación y el análisis de las normas de convivencia socioculturales de esta segunda lengua con el fin de dar una imagen adecuada en las relaciones profesionales" (ANECA, 2004). Sin embargo, somos conscientes de que el desarrollo de las competencias profesionales y de un lenguaje especializado se adquiere mucho mejor, en nuestra modesta opinión, en una etapa posterior a la adquisición de la lengua denominada "común", de manera que consideramos necesaria una etapa previa de adquisición de la lengua común para, posteriormente, en los dos últimos años del Grado, adquirir los conocimientos profesionales.

La importancia de una segunda lengua extranjera común no se contempla claramente en los nuevos planes de estudios, centrados fundamentalmente en la adquisición de conocimientos profesionales, pero quedó manifiestamente respaldada por numerosos representantes de los principales sectores de la industria turística en el I Congreso Internacional de Turismo y Lenguas Extranjeras (Palma de Mallorca, abril 2011) pues se llegó a señalar que, en muchas ocasiones, preferían contratar simplemente a personal nativo porque la formación posterior en conocimientos profesionales era más rentable para la empresa y que, por otro lado, se necesita a profesionales que sepan comunicar aspectos amplios y cotidianos, dado que muchas veces se habla de aspectos de la vida cotidianos (como, por ejemplo, el tiempo o el retraso que ha tenido el vuelo).

De hecho, observamos que este aspecto se refleja nítidamente en el Libro Blanco, pues presenta en la adquisición de Conocimientos Profesionales ("Saber hacer") situaciones de comunicación "habituales y personales". Pensamos por consiguiente que se intenta resaltar la importancia de la adquisición de la lengua común, pero, como se aprecia, se incide bastante más en los conocimientos profesionales:

a) Comprender y producir mensajes orales en situaciones diversas de comunicación: habituales y personales.

b) Interpretar y realizar información escrita tanto en el ámbito turístico como en el ambiente sociocultural.

c) Comunicarse de forma oral y escrita con un interlocutor, interpretando y transmitiendo la información necesaria para establecer los términos que delimiten una relación profesional dentro del sector turístico.

d) Analizar las normas de convivencia socioculturales con el fin de dar una imagen adecuada en las relaciones personales y profesionales. (ANECA, 2004).

No parece demasiado arriesgado asegurar que las asignaturas que demandan mayor grado de presencialidad por parte del alumno y un mayor porcentaje de la ya casi obsoleta "lección magistral" dentro del Grado en Turismo parecen ser los idiomas; el estudiante -en especial el más débil en relación a la oralidad de la lengua- precisa de un sistemático aporte de la segunda lengua que no siempre puede verse satisfecha con los nuevos modelos de tutoría, sobre todo si entendemos ésta como de grupo o seminario y, además, rotativa, aunque ésta favorezca por otra parte el aprendizaje colaborativo. Santos Guerra (1999), de la Universidad de Málaga, escribía ya incluso antes de la materialización del proceso de Bolonia en nuestro país — de forma casi profética - acerca de la problemática subyacente de toda universidad a la hora de facilitar los procesos de enseñanza y aprendizaje. Las condiciones organizativas de los estudios superiores obstaculizan la evaluación fiel y de calidad, ya sea por masificación de alumnos o no:

La masificación de alumnos dificulta la tarea de hacer evaluación rigurosa. Digo dificulta, no impide, porque algunos profesores hacen lo mismo con 5 que con 500.

Un pequeño grupo de alumnos permitiría al profesor conocer cómo aprende cada uno, qué sabe, qué dificultades tiene para el aprendizaje. Lo que hace cotidianamente en el aula serviría como referente fundamental para su aprendizaje. 
Los profesores explican oralmente y casi siempre preguntan por escrito. ¿Cómo van a disponer de tiempo para hacer entrevistas individuales? Es el sistema lo que lo impide (Santos Guerra, 1999, p. 379-80)

¿Han resuelto estos problemas los nuevos modelos de reciente implante? Quizás. Lo que parece claro es que los grandes beneficiados del nuevo sistema de aprendizaje por competencias y PATU (Plan de Acción Tutorial Universitaria) son los alumnos “virtuales", los eternos ausentes, los nombres en lista, para quienes la modalidad no presencial o semi-presencial ha supuesto el fin de sus cuitas con ese porcentaje elevado dedicado al examen final que, con todo, ha de ser superado para lograr el paso a la evaluación de la destreza de producción oral.

Por ahora, y antes de afrontar este aspecto, dedicaremos las próximas líneas a incidir en la repercusión que el Espacio Europeo de Educación Superior ha tenido en la docencia y el aprendizaje de lo que más adelante conoceremos como "Lenguas de Especialidad", o, lo que es lo mismo y nos compete en esta ocasión: las asignaturas de idiomas extranjeros en el Grado en Turismo.

\section{Implicaciones del espacio europeo de educación superior en el aprendizaje de lenguas extranjeras}

Trasladar el desarrollo de la interacción comunicativa al aula significa tener que plantearse, y llevar a la práctica, nociones importantes en el actual escenario de enseñanza universitaria. La autonomía del aprendiz, las metodologías activas y el aprendizaje cooperativo son - ya lo hemos adelantadoconceptos ya nada novedosos en nuestros días y aceptados por la comunidad universitaria, pero con una razón de ser significativa en el marco en el que nos encontramos:

[e] tipo de aprendizaje que se realiza en la Enseñanza Superior debería concordar realmente con la idea de superior, en la medida en que se refiere a un estado mental que se halla por encima de la receta convencional del aprendizaje (...) [u]na forma muy relevante de proporcionar el carácter superior al aprendizaje universitario es (...) potenciar el grado de autonomía personal e intelectual del estudiante (Rué, 2009, p.97).

El planteamiento de estos conceptos aproxima a una metodología basada en el Proyecto de Aprendizaje Tutorado (PAT), estrategia docente de larga tradición en el ámbito anglosajón, asentada principalmente en el aprendizaje independiente de los estudiantes y en el seguimiento de este aprendizaje por parte del profesor-tutor.

Las características más relevantes del PAT son detalladas por Álvarez Rojo, García Jiménez, Gil Flores, y Romero (2004: 24).

- Oferta docente diseñada para promover el aprendizaje autónomo 3 de los estudiantes, bajo la tutela del profesor y en escenarios variados (académicos y profesionales).

- Oferta de enseñanza prioritariamente referida al aprendizaje del "cómo hacer las cosas" (know how).

- Oferta de enseñanza basada en la asunción por los estudiantes de la responsabilidad sobre su propio aprendizaje (aprendizaje independiente).

La metodología del PAT introduce cambios tanto en las metas como en el proceso de aprendizaje pues el objetivo es dotar al alumno de herramientas, enseñarles a aprender. Es, en definitiva, una metodología de enseñanza-aprendizaje enfocada a "aprender a aprender", a desarrollar capacidades o competencias presentándoles problemas reales, el saber-hacer, en los que confluyan perspectivas disciplinarias complementarias.

El planteamiento del PAT se adecúa a la enseñanza-aprendizaje de idiomas extranjeros, pero conlleva cambios importantes. Uno de ellos es la búsqueda del equilibrio entre el conocimiento conceptual y

\footnotetext{
3 En este proceso, toma gran relevancia el aprendizaje autónomo, pero conviene resaltar la diferencia entre aprendizaje independiente (aprender algo por su cuenta, al margen de todo reconocimiento y marco institucional, a la manera autodidacta) y autonomía como estrategia docente en el Espacio Superior, como método y como fin: "[t]odo alumno puede aspirar a alcanzar mayores cuotas de autonomía mediante la formación" (Rué, 2009, p 87-88).
} 
el conocimiento procedimental; la formación universitaria a menudo tiende a un enfoque demasiado conceptual donde prima la gramática y el léxico mientras que las habilidades discursivas y las estrategias que implica todo conocimiento reciben menos atención en las clases. Otro cambio significativo es que además de "enseñar", es decir, transmitir conocimiento, con el PAT debemos enseñar a "hacer las cosas" y "enseñarles a aprender" para que puedan llevar a cabo un aprendizaje autónomo. Pensar en aprendizaje autónomo en lenguas extranjeras puede resultar inconcebible pues la mentalidad tradicional hace casi impensable el aprendizaje de un idioma sin el profesor. Sin embargo, la adquisición de una lengua extranjera no acaba en el aula, más bien es en ella donde empieza dado que ahí es donde les proporcionamos las herramientas para que puedan aprender por sí solos. En este sentido, hay que destacar que esta metodología propia del "aprender a aprender" se ha visto claramente reforzada en el aprendizaje de idiomas por el potencial de las nuevas tecnologías y el uso de las Tecnologías de la Información y la Comunicación (TIC's). Las TIC's las podemos utilizar antes, durante y después de la clase. Fuera del aula, para ofrecer contenidos de profundización y posibilitar la comunicación entre profesor-alumno y alumno-alumno, fomentando, de esta manera, el aprendizaje cooperativo.

Además de todos los valores del PAT, esta metodología es doblemente recomendable, pues el estudiante logra algunas de las competencias que todo universitario deberá adquirir y que son demandadas en el Grado en Turismo: las competencias trasversales. Según establece el Real Decreto que regula los estudios oficiales de grado, "los estudios universitarios deberán proporcionar a los alumnos la formación que aúne: conocimientos generales básicos y conocimientos transversales relacionados con la formación integral de la persona; y conocimientos y capacidades específicas" (Benito y Cruz, 2005, p. 14). Así, la distribución de las competencias quedaría enmarcada del siguiente modo:

\section{Instrumentales}

- Capacidad de análisis y de síntesis

- Capacidad de organización y planificación

- Capacidad de observar fenómenos lingüísticos

- Conocimiento de una lengua extranjera

- Conocimientos informáticos relativos al ámbito de estudio

- Capacidad de gestión de la información

- Resolución de problemas

- Toma de decisiones

\section{Personales}

- Trabajo en equipo

- Reconocimiento de la diversidad y multiculturalidad

- Razonamiento crítico

- Responsabilidad

- Confianza en sí mismo

- Capacidad de hacer y recibir críticas

- Capacidad de toma de decisiones

- Compromiso ético

- Trabajo en equipo

\section{Sistémicas}

- Aprendizaje autónomo 
- Adaptación a nuevas situaciones

- Creatividad

- Conocimiento de otras culturas

- Motivación por la calidad

- Iniciativa

Para el docente en lenguas extranjeras, los cambios surgidos en estos años en la Enseñanza Superior reflejan la relevancia que posee el grado de actuación del discente en su formación del saber-hacer profesional. En el caso del graduado en Turismo prevalece el control de las lenguas extranjeras en su uso socio-profesional, lo cual implica desarrollar las competencias en lenguas extranjeras al tiempo que se impulsa un saber-hacer profesional. El desarrollo de éste traslada a la adquisición de actos de comunicación específicos de turismo como saludar, dar la bienvenida, atender al cliente en sus necesidades y deseos, responder de manera educada a reclamaciones y quejas acerca del servicio recibido, saber presentar monumentos en una visita guiada, etc. Engloba, en definitiva, saber utilizar en el mundo profesional turístico una lengua extranjera adecuada y adaptada a cualquier contexto social y cultural que se pueda producir.

\section{El aprendizaje de idiomas en el sector turístico: lengua de especialidad}

El Profesor Emérito Pierre Lerat realizó en 1995 una interesante reflexión sobre las Lenguas de Especialidad que no puede ser obviada en las páginas que nos ocupan. Según él, este tipo de lenguas se plantean escasamente desde el punto de vista de las preocupaciones de la teoría lingüística (1995, p. 11), de ahí que entre los objetivos del estudioso esté redefinir el concepto de Lengua de Especialidad (de aquí en adelante frecuentemente denominada “LE”). De acuerdo con su opinión, la concepción que algunos estudiosos tienen de este concepto como un sub-sistema o sub-lengua autónoma es errónea. Para el lingüista, la LE es, en el caso del francés, "c'est tout à fait du français et en même temps c'est le vecteur de savoirs et de savoirs-faire” (Lerat, 1995 p. 13).

La misma opinión de Lerat es compartida igualmente por el prestigioso lingüista Émile Benveniste, quien, en la línea del anterior autor, escribió ya más de viente años antes: "Ce qui change dans la langue, ce que les hommes peuvent changer, ce sont les désignations, qui se multiplient, qui se remplacent et qui sont toujours conscientes, mais jamais le système fondamental de la langue" (Benveniste, 1974, p. 94).

Analizando las aportaciones de estos escritores, podemos afirmar que hablar de Lenguas para (o con) Fines Específicos significa considerar que se lleva a cabo un uso especial de las mismas reglas y convenciones de la lengua general; se trata, de nuevo en palabras de Lerat, del uso de una lengua natural para dar cuenta técnicamente de conocimientos especializados (ibid.: 21) y, por lo tanto, desde el punto de vista de la enseñanza, no se debe limitar a un enfoque lexicológico sino que debe ser una perspectiva centrada en los aspectos comunicativos y discursivos, pues, como Sager et al. (1980, p. 52) arguyen, las LE son subconjuntos del lenguaje fundamentalmente pragmáticos.

La LE presenta, por ende, una ventaja evidente: remite al sistema lingüístico para la expresión y a las profesiones para los saberes - "renvoyer au système linguistique pour l'expression et aux professions pour les savoirs” (Lerat, 1995, p. 13).

Es más que sabido que, en la sociedad actual, las necesidades profesionales requieren cada vez más de unos conocimientos lingüísticos especializados. Con relación al ámbito del saber y formación que nos ocupa, la difusión de la lengua del turismo en el campo de la enseñanza de las lenguas extranjeras y en la traducción nos lleva a considerar la lengua del turismo como una LE que no se limita a la mera transmisión de conocimientos entre profesionales; al contrario, la interpretación de LE del turismo ha de ampliarse hacia el punto de vista de la comunicación entre profesionales y usuarios/clientes. Es más: para Mangiante y Parpette, la expresión Lengua de Especialidad tiene sus limitaciones desde el punto de vista didáctico, pues "une discipline n'existe pas à travers une langue, qui serait homogène, mais à travers des discours extrêmement divers: écrits ou oraux, spontanés ou 
distanciés, sous forme d'exposé ou d'échange dialogué, d'article spécialisé, de fiche technique, etc." (Mangiante y Parpette, 2004, p. 18).

El riesgo de considerar una LE, repetimos, está en que en la mayoría de los casos se tiende a centrar la atención en el léxico específico, ignorando el aprendizaje de otras competencias: "les discours sont en effet beaucoup plus façonnés par les paramètres de la situation de communication que par les contenus véhiculés" (Mangiante y Parpette, 2004, 2004, p. 19).

Una vez analizado el significado de LE, es conveniente señalar que, en la enseñanza de lenguas extranjeras, algunos autores establecen una división entre Lengua de Especialidad y Lengua con Objetivos Específicos. En efecto, se considera que el primero, tal como su nombre indica, se centra en la especialidad, es un acercamiento a una rama de actividad profesional, está abierto a un público lo más amplio posible e intenta dar cuenta de la diversidad del campo. Así, los métodos del francés, inglés o alemán del turismo, por ejemplo, abordan diferentes oficios: hostelería, organización de viajes, visitas guiadas, restauración, etc. En cambio, la lengua con objetivos específicos cubre todas las situaciones -estén o no ancladas en una especialidad- y se caracteriza por su manera de enfocar el aprendizaje trabajando caso a caso, oficio a oficio, en función de las necesidades de un público concreto (Mangiante y Parpette, 2004, pp. 16-17). Su característica principal es la precisión del objetivo que se desea conseguir y la urgencia de este objetivo (Mangiante y Parpette, 2004, p. 21), orientando la enseñanza sólo hacia aquellas situaciones de comunicación con las que se enfrentará el estudiante en su actividad profesional. Aunque la frontera entre uno y otro no sea muy rígida, ya que un programa a medida puede coincidir más o menos con el contenido de un material que existe en el mercado, conviene tener en cuenta esta distinción en la enseñanza de lenguas extranjeras en el turismo, ya que cuanto más clara sea la demanda, más evidentes serán para el docente las necesidades específicas y las situaciones de comunicación.

Así pues, como hemos visto, la lengua que se utiliza en el ámbito turístico puede efectivamente considerarse una LE, que no es más que una lengua "natural" que refleja conocimientos especializados. Estamos ante una variedad funcional de la lengua común, es decir que presenta algunos rasgos de ella, pero, a su vez, adquiere una terminología propia y unos rasgos lingüísticos, pragmáticos y funcionales propios.

El discurso de una LE se centra más que ninguna otra en el saber-hacer, idea muy en consonancia con los nuevos planteamientos de la enseñanza universitaria y competencia estrechamente vinculada con los postulados recogidos en nuestras líneas.

Es indudable, así hemos concluido, que la LE presenta connotaciones didácticas y metodológicas importantes, pues se deberá primar la orientación de los objetivos al aprendizaje de situaciones propias del sector. En este sentido, es de suma trascendencia el conocimiento que los docentes de lenguas extranjeras en Grado en Turismo tengan del contexto profesional específico, pues una formación en LE implica un estudio en profundidad de las condiciones en las que los aprendices deberán emplear esta lengua ya como vehículo de comunicación.

Como hemos visto, el desarrollo de las competencias profesionales de nuestros discentes dependerá en gran medida de esta formación por parte del profesor y de la exposición que éste desarrolle de la LE no sólo en el ámbito lexicológico o gramatical sino también interactivo y de comunicación. Hora es ya de adentrarnos en la labor de interactuación, de producción oral, con un análisis somero de las complejidades que le son inherentes.

Interacción comunicativa en el aula de segundas lenguas del grado en turismo: dificultades psicolingüísticas del aprendizaje por competencias

En líneas previas hemos venido incidiendo en cómo la nueva metodología pretende logros en relación a la independencia académica de los alumnos, su motivación investigadora o su individualidad en la gestión bibliográfica y formativa. El profesor no se manifiesta ya como epicentro del proceso educativo, sino como orientador, coordinador o facilitador del aprendizaje, provocando la intervención del discente en la toma de decisiones y apelando a su capacidad autónoma. 
El modelo organizativo de orientación universitaria y de acción tutorial persigue la adquisición de unas competencias que, como postula Cano González (2009, p.186), ahora "son un requisito" y no una "opción". No entraremos a valorar la carencia de potestad individualista que, por lo general, caracteriza al alumnado de las últimas generaciones, pero sí habría que tasar la valía de las estrategias que empleamos para alcanzar nuestros objetivos en segundas lenguas. Se pretende el entrenamiento de los estudiantes de Turismo para ser capaces de trabajar en un contexto internacional, potenciar sus habilidades en las relaciones interpersonales y enfocar su formación hacia un claro reconocimiento a la diversidad y la multiculturalidad.

Además de estas competencias de carácter personal y las ya muy señaladas de índole sistémica aprendizaje autónomo, conocimiento de otras culturas y costumbres o la motivación por la calidad, el Libro Blanco del Grado en Turismo insiste en la obtención de otras tantas instrumentales, entre las que figuran:
a) Manejar técnicas de comunicación
b) Trabajar en Inglés como lengua extranjera
c) Comunicarse de forma oral y escrita en una segunda lengua extranjera
d) Comunicarse de forma oral y escrita en una tercera lengua extranjera

Si bien los estudios de Turismo están caracterizados por su naturaleza multidisciplinar y transdisciplinar y la propia especificidad del título hace que su desarrollo profesional implique el enfrentamiento a los problemas relacionados con la gestión de personas, empresas e instituciones -así lo estipula Libro Blanco-, en términos generales los alumnos del Grado lamentan no disponer de una mayor exposición a los idiomas extranjeros —disciplinas que, además, suelen ser de su agrado y tienden a valorar como imprescindibles dentro del marco de la titulación-. Por otra parte, es una más que dura realidad que el dominio de idiomas de los alumnos españoles se aleja en gran medida del marco social y educativo de otras naciones europeas ${ }^{4}$, de ahí que fomentar su uso competente sea vital.

En relación a este último punto podemos afirmar lo que es casi una obviedad: el estudio de una segunda lengua - ya sea con fines específicos, como es el caso que nos ocupa en este artículo, ya con un objetivo meramente instrumental- persigue una meta evidente: ser capaces de dominarla de manera fluida, emplearla como vehículo de interacción comunicativa para la expresión y transmisión de pensamientos, sentimientos, ideas, etc. Apunta R. Schmidt (1992) que la fluidez no es sino la consecuencia de la automatización del lenguaje y de la madurez en los procesos psicolingüísticos de planificación y producción del lenguaje. A la opinión de este exegeta añadiremos nosotros que lograr este desarrollo dependerá en gran medida de su exposición a coyunturas que lo arrastren a la práctica de su fluidez en el empleo del idioma y que hagan que ésta sea valorada antes que su exactitud y corrección lingüística. Atenderemos en breve a esta particularidad.

Es más que conocido que, en el marco de la docencia de idiomas extranjeros, las metodologías más recientes abogan por un marcado perfil interactivo; la función comunicativa es fundamental en las lenguas de especialidad, ya lo hemos dicho, de manera que debemos tener presentes los elementos fraseológicos, sintácticos, estilísticos, etc., indisociables todos ellos de toda función de expresión y de comunicación. Ni qué decir tiene que todos los componentes de la lengua entran a formar parte del proceso de comunicación, pues ésta no se limita a los intercambios orales: conscientes somos de que existe comunicación asimismo cuando leemos un texto o cuando escribimos una carta o una nota, también cuando escuchamos una grabación.

Si bien nuestros educandos de Grado en Turismo deberán desarrollar ambas formas de transmisión informativa, este epígrafe desea presentar exclusivamente el planteamiento llevado a cabo en el proceso de la expresión oral, pues se nos figura ésta una destreza poco valorada en el aprendizaje de lenguas extranjeras. Nuestra experiencia nos ha llevado a deliberar sobre nuestros propios modelos

\footnotetext{
4 Pavón Vázquez (2009, p. 66) carga las tintas en este punto recuperando índices del Eurobarómetro del 2005 (Comisión Europea). De acuerdo con estos datos, de entre los 25 países miembros de la Unión Europea, España se sitúa en el puesto 21.
} 
de enseñanza, a cuestionarnos si la práctica de la expresión oral que efectuamos en el aula es realmente un modo de interacción adecuado o si las limitaciones con las que copamos a diario académicas, presupuestarias ${ }^{5}$, etc.- están afectando seriamente a nuestra labor y a la consecución de las competencias detalladas en las guías docentes de nuestras asignaturas, algunas de las que ya hemos dado cuenta en páginas anteriores.

Los futuros profesionales de la industria turística se sienten especialmente sensibilizados al respecto del entrenamiento y desarrollo exitoso de la destreza de producción oral, "la destreza —como considera Mateo- de mayor complejidad didáctica ya que las situaciones de habla que se crean en el aula son, de nuevo y casi siempre, ficticias aunque traten de reproducir la realidad" (Mateo, 1999, p. 184). Los alumnos no restan importancia a la variante escrita de la lengua —la problemática de la producción escrita bien merecería un artículo independiente a éste-, pero reconocen en la retórica de su profesión una función básica e indispensable, máxime cuando ésta ha de ser desarrollada en la que no es su lengua materna.

El contexto turístico, por otra parte, presenta una evidente y marcada función de atención social no en vano forma parte del denominado "Sector Servicios"-, lo cual, a su vez, conlleva una indudable sensibilización por el contexto lingüístico situacional, la pragmática dialogística —con todo lo que ésta supone: adecuación del registro lingüístico empleado, modelos de comportamiento social (cortesía verbal, valores cívicos, tabúes dependientes del grupo en cuestión, etc.)—o incluso variables relativas al lenguaje no verbal — proxémica, kinésica, etc.—. No conviene olvidar que el devenir del enunciado oral habrá indiscutiblemente de verse sujeto a un equilibrio coyuntural que evite situaciones incómodas, malentendidos, etc.

Esta vertiente, a medio camino entre lo sociológico y lo lingüístico, acarrea asimismo una visión psicológica: la preocupación por el alumno a ser observado, escuchado y/o evaluado por sus compañeros o por el propio profesor durante el ejercicio de oralidad es, sin lugar a dudas, una fuente de aprensión que atenta contra la consecución de algunas competencias, lo que podríamos considerar casi un "efecto secundario" derivado del aprendizaje de una lengua extranjera. Ortega Cebreros (2003) ha estudiado en profundidad el factor psicológico de la ansiedad, que, a todas luces, afecta al producto final en nuestras asignaturas de idiomas:

Having students that perceive considerable levels of anxiety in the classroom has been regarded as an experience that is more likely to occur in foreign language lessons than in lessons on other subjects of the curriculum. This course of events seems natural if we take into account that in foreign language classrooms the students have to cope with the demands of being able to sustain communication by means of an instrument they are not completely familiar with. (...) University students with an extensive language learning background can also perceive considerable levels of language anxiety. (Ortega Cebreros, 2003, p. 1)

No es baladí afirmar que toda metodología activa de índole oral que de aquí en adelante emprendamos requerirá de una doble articulación: por una parte, demandará la participación de un perfil estudiantil extrovertido, abiertamente dispuesto, pues la práctica oral implica cierta mezcolanza de complejidades diversas ${ }^{6}$ :

1. La inmediatez de la demanda de producción:

\footnotetext{
5 El momento universitario actual es todo un grito desoído, una sublevación del sector docente y estudiantil por los recortes económicos que están derivando en la supresión de ciertas asignaturas fundamentales en el currículo de las titulaciones, la unificación de grupos que dificultan la impartición correcta de algunas materias o la imposibilidad de contratación de profesorado experto.

${ }^{6}$ La visión de Rubio Alcalá se nos antoja tremendista y poco alentadora cuando, siguiendo las teorías de Guiora (2004), señala que el método comunicativo es una "proposición psicológica desconcertante" que incluso puede llegar a reducir el nivel de autoestima, disminuir la confianza del alumno, generar inhibición, restringir su participación en el aula y acotar las posibilidades de buenos resultados en los procesos de evaluación (pp. 82-3). ¿Qué sentido tendría la metodología comunicativa si la contemplásemos bajo una perspectiva tan nociva y desventajosa? Y, lo que es más, ¿cómo alcanzaría el alumno la madurez interactiva si ésta no se potenciase en el aula de segundas lenguas? No pretendemos obviar la dificultad que los estudiantes menos directos habrán de afrontar en estas lides, pero tampoco podemos tildar a una destreza de tan evidente trascendencia de perjudicial y arriesgada.
} 
Este tipo de práctica puede no ir acompañada de un intervalo de preparación y tratar de promover la capacidad de expresión simultánea y presta del alumno en la L2. Esto conllevará errores lógicos por transferencia, calcos de la lengua materna, o, como Mateo advierte ingeniosamente, "habla prefabricada", es decir, el empleo de fragmentos del idioma de corte convencional que almacenamos en nuestra mente y que aprovechamos para evitar una selección paradigmática y sintagmática desde cero. . (Ortega Cebreros, 2003, p 185)

2. Restricciones de orden lingüístico y funcional:

Aunque comúnmente la tarea de producción oral persigue la fluidez antes que la precisión, el estudiante suele inquietarse por tratar de emplear la estructura sintáctica adecuada, el léxico idóneo y la modulación fonético-fonológica que más se asemeja a la de la lengua extranjera. Mateo vuelve a estar en lo cierto cuando asegura que el intento del alumno por expresar de forma sencilla sus enunciados acaba por tornarse en dificultad por razones de polisemia, implicaciones pragmáticas y conocimiento textual y compartido (Guiora,2004, p.184).

3. Elementos de refuerzo de la conversación de carácter fático y supralingüístico:

Entendidos como apoyaturas que sobrepasan la mera expresión de ideas para alcanzar un nivel más profundo que se sensibiliza por la atención del interlocutor y su reacción al enunciado emitido.

4. Mantenimiento del ritmo de la expresión:

O fluidez, concepto que no ha de ser confundido con el de "velocidad", pues el último se circunscribe a parámetros de índole temporal en la exposición, mientras que el primero hace referencia a la preservación de un equilibrio adecuado en el flujo de la enunciación.

Por otra parte, el rol motivador del profesor habrá de hacer sentir al alumno parte de la comunidad académica, subrayar la relevancia que en el mundo turístico tiene el hacerse comprender en una lengua extranjera y propugnar el desarrollo del conocimiento de la disciplina, alimentando en el discente — también en aquel retraído e indeciso- la necesidad de interacción ${ }^{7}$, aun cuando ésta acarree el riesgo de fluctuación en el manejo de los recursos retóricos de la L2. La aprensión comunicativa, no lo olvidemos, es un variante de la ansiedad consecuencia del miedo a producir un acto comunicativo, pero ha de entenderse como parte del proceso de aprendizaje y no permitir que ésta obstaculice en demasía la oportunidad de que los errores en la oralidad sean corregidos de forma constructiva (véase Brown, 1996).

Littlewood también analiza en detalle esta limitación, íntimamente vinculada a la dimensión afectiva del alumno. Para el autor, la práctica oral en el aula puede implicar un sentimiento de alienación, pues el estudiante se ve abocado al re-aprendizaje de contextos situacionales de su día a día que, muy frecuentemente, le resultan obvios y, por ende, casi absurdos:

Unless they have firm confidence in themselves, they may come to feel that they project a silly, boring image and become withdrawn. Their sense of alienation may be increased by the fact that they are having to re-learn the conventions which surround simple daily events, such as eating in a restaurant or approaching an acquaintance. To use two terms commonly applied to this kind of experience: they may develop a sense of "reduced personality" and experience various degrees of "culture shock". (Littlewood, 1981, p. 16)

7 Añade Mateo (1999) a este respecto:

El profesor debe adoptar una actitud generosa hacia los esfuerzos de los educandos, ofrecerles tareas interesantes y con unos fines claros; debe mantener un cierto grado de disciplina que, sin coartar la libertad del alumno, estimule el trabajo organizado y en calma; debe tratar de mostrar siempre el mismo interés y ánimo, aunque en muchas ocasiones le resulte una actividad rutinaria; debe alentar la interrelación de los estudiantes y una voluntad cooperadora por medio del trabajo en grupo y colectivo; debe desarrollar en los alumnos la capacidad de la autocrítica y autovaloración de su trabajo y debe animarlos en sus éxitos sin ser demasiado crítico en sus fracasos. (p. 77) 
Sea como fuere, y a pesar de la complejidad que el aprendizaje de la destreza oral puede tener, la enseñanza por competencias en la titulación de Turismo sigue marcando como imprescindible el manejo de segundas lenguas. Entre los conocimientos profesionales se demanda del alumno la capacidad de comunicación en cualquier medio, el empleo de la lengua en todas sus vertientes en un entorno profesional y la transmisión de información con capacidad pragmática para interpretar datos necesarios dentro del marco turístico.

La oralidad de la lengua, como perfil indisociable de un idioma, requerirá, como hemos visto, de la competencia gramatical, sociolingüística, discursiva y estratégica — siguiendo el modelo de subcompetencias diseñado por Canale allá por los años 80- para su adecuación a las normas de convivencia socioculturales de la lengua que se hable y para, como puede leerse en el Libro Blanco (ANECA, 2004, p.132) y ya hemos comentado, "dar una imagen adecuada de las relaciones profesionales" del sector turístico. Es nuestra competencia —no podríamos emplear un término más significativo- guiar al alumno hacia el éxito en estos objetivos, aun cuando hayamos de superar limitaciones de corte psicosociolingüístico como las que hemos analizado que, en ocasiones, conviertan el aprendizaje por competencias en una compleja labor.

\section{Conclusiones}

El análisis llevado a cabo de las implicaciones del EEES en los estudios de Grado en Turismo y sus repercusiones en la adquisición de lenguas extranjeras nos ha permitido tener una visión de conjunto sobre el proceso de adaptación, el porqué de dicho proceso y las repercusiones en el aprendizaje de lenguas extranjeras.

Queda patente con lo planteado que la evolución de la sociedad, y del turismo más concretamente, demanda profesionales con una formación específica y diferenciada en el sector, una de las razones por la que era más que justificada la adaptación de los planes de estudios universitarios. De esta adecuación hacia la formación de profesionales especializados nacen los estudios de grado, y en el caso que nos ocupa, el Grado en Turismo, formación superior que ambiciona una adaptación continua a las nuevas demandas del sector.

Es precisamente la influencia de este requerimiento de adaptación a las evoluciones del sector la que nos lleva a resaltar la importancia de considerar los idiomas extranjeros enseñados en el Grado en Turismo, como lenguas, sí, pero lenguas de especialidad, que, contempladas bajo el prisma de Lerat (1974, p. 21) son, en definitiva, lenguas naturales que dan cuenta técnicamente de conocimientos especializados.

Consecuentemente, la enseñanza de lenguas extranjeras en el Grado en Turismo experimenta cambios. Por un lado, porque se ve afectada por el nuevo marco de Enseñanza Superior; por otro, porque es labor de los docentes de lenguas extranjeras, de lenguas de especialidad, conocer las necesidades profesionales y lingüísticas particulares del sector para así orientar el aprendizaje. El conjunto implica, como se ha visto a lo largo del artículo, que los docentes adoptemos una nueva metodología con unas características destacables en las que prime la autonomía del discente y se forme en competencias (tanto personales como sistémicas o instrumentales).

Estas dos características señaladas son compatibles con la enseñanza de lenguas de especialidad. Tanto la autonomía en el aprendizaje de lenguas extranjeras como la formación en competencias no están suponiendo grandes problemas para nuestros estudiantes. Contamos con un elemento importante: su gran motivación inicial dado que su promoción profesional está unida a este aprendizaje. Gracias al refuerzo de los Proyectos de Aprendizaje Tutorado preparamos a nuestros estudiantes a tener iniciativa, a emprender cualquier cosa nueva, a potenciar sus habilidades en las relaciones interpersonales, a responder a los retos de la época. No ocultamos sin embargo que han existido ciertas reticencias derivadas de la carga de trabajo que supone esta metodología, pero en general el estudiante se siente motivado y activo, se implica más y es más participativo. Le agrada bastante desarrollar tareas con cierto grado de dificultad, relacionadas con el ámbito del turismo, y considera que "aprende más". 
Como docentes en lenguas extranjeras, este periodo de transformaciones nos ha llevado a sensibilizarnos hacia una de las destrezas de mayor complejidad didáctica, a menudo relegada a un segundo plano - bien sea por motivos de tiempo o porque los grupos numerosos en las aulas imposibilita su desarrollo- pero de gran importancia en el contexto turístico: la producción oral. En un entorno, como es el turístico, en el que priman las relaciones profesionales, el trabajo de la oralidad de las lenguas de especialidad implica el desarrollo de la competencia gramatical, sociolingüística, discursiva y estratégica, pero también hemos querido destacar la superación de las nada desdeñables limitaciones de orden psicosociolinguístico.

Como conclusión, señalemos que nuestra experiencia nos permite afirmar que la conjunción de todos estos aspectos requieren una mayor atención por parte del docente hacia el proceso de enseñanza-aprendizaje que la basada en la mera transmisión y acumulación de información; en el caso de las lenguas de especialidad, la transición del mero conocimiento conceptual (gramática y léxico) al procedimental (competencias discursivas, estratégicas) que les permita desarrollar el saber-hacer profesional. Destacamos igualmente el esfuerzo, sobre todo en los primeros años de estudios universitarios, para que sepan gestionar su aprendizaje autónomo. Conscientes de esta dificultad, optamos por proporcionarles un trabajo muy bien organizado y secuenciado, guiándoles en su propio aprendizaje, pero permitiendo que el aprendiz trabaje a su ritmo y sabiendo dónde focalizar sus esfuerzos según sus puntos débiles.

La adaptación de modelos pedagógicos que fomentan la autonomía del estudiante en la enseñanza de lenguas extranjeras nos pone en consonancia con la acertada manifestación de Ernesto Martín Peris. Como docentes de lenguas de especialidad sentimos que "el alumno es quien actúa y yo puedo abrirle ventanas, ventanas a la lengua y al aprendizaje” (Martínez Gila, 2011, p. 7).

\section{Bibliografía}

Agencia Nacional De Evaluación de la Calidad y Acreditación (ANECA). (2004). Libro Blanco del Título de Grado en Turismo.

Álvarez Rojo, V. Álvarez Rojo, V., García Jiménez, E., Gil Flores, J. y Romero Rodríguez, S (2004). La enseñanza universitaria: planificación y desarrollo de la docencia. Madrid: Eos.

Benito, A. y Cruz, A. (2005). Nuevas claves para la Docencia Universitaria en el Espacio Europeo de Educación Superior. Madrid: Narcea.

Benveniste, E. (1974). Problèmes de linguistique générale, 2. Paris: Gallimard.

Bernal Aguado, J. L. (2006). "Diseño curricular en la enseñanza universitaria desde la perspectiva de Ios ECTS. Pautas para el diseño de una asignatura”. Universidad de Zaragoza. (Enlace web: http://www.unizar.es/eees/doc/pautas_ects.pdf). Consultado en fecha (23-12-2012)

Brown, B. (1996). Speakers, Listeners and Communication: Explorations in Discourse Analysis. Cambridge: Cambridge University Press.

Canale, M. (1983). From communicative competence to communicative language pedagogy. En J.C. Richards y R. W. Schmidt, (Eds.), Language and Communication. Londres: Longman.

Cano González, R. (2009). “Tutoría universitaria y aprendizaje por competencias. ¿Cómo lograrlo?” En Revista Electrónica Interuniversitaria de Formación del Profesorado. 12 (1), pp. 181-204.

Lerat, P. (1995). Les langues spécialisées. Paris: Presses Universitaires de France.

Littlewood, W. (1981). Communicative Language Teaching: An Introduction. Nueva York: Cambridge University Press.

Mangiante, J.M. y Parpette, C. (2004). Le Français sur Objectif Spécifique: de l'analyse des besoins à l'élaboration d'un cours. Paris: Hachette.

Martínez Gila, P. (2011). “Entrevista con Ernesto Martín Peris. Primer director académico del Instituto Cervantes". Marco ELE. Revista de didáctica Español como Lengua Extranjera. (Enlace web: http://marcoele.com/descargas/12/entrevista-martin.pdf). Consultado en fecha (4-10-2012). 
Mateo Martínez, J. (1999). La enseñanza universitaria de las lenguas extranjeras. Alicante: Servicio de Publicaciones de la Universidad de Alicante.

Ortega Cebreros, A. M. (2003). "Measuring Language Anxiety Perceived by Spanish University Students of English". Bells: Barcelona English Language and Literary Studies, 12. (Enlace web: http://www.publicacions.ub.edu/revistes/bells12/PDF/art11.pdf). Consultado en fecha (15-10-2012)

Pavón Vázquez, V. (2009). "Cambios en la realidad educativa a través de la enseñanza integrada de lengua y contenidos”. En M.T. Pedraz (coord.), Las lenguas extranjeras como vehículo de comunicación intercultural (pp. 65-83). Madrid: Ministerio de Educación. Serie Humanidades.

Rubio Alcalá, F. D. (2004). La ansiedad en el aprendizaje de idiomas. Huelva: Servicio de Publicaciones de la Universidad de Huelva.

Sager, J.C., Dungworth, D., Mcdonald, P. (1980). English Special Language. Principles and Practice in Science and Technology. Wiesbaden: Brandstetter.

Slagter, P. (1979). Un Nivel Umbral. Estrasburgo: Publicaciones del Consejo de Europa.

Schmidt, R. (1992). "Psychological Mechanisms Underlying Second Language Fluency". Studies in Second Language Acquisition, 14: 357-85

Van Ek, J. A. (1975). The Threshold Level in a European Unit/Credit System for Modern Language Learning by Adults. Estrasburgo: Publicaciones del Consejo de Europa. 


\section{Autores}

María del Pilar Blanco Calvo

Es Diplomada en Educación, Licenciada en Filología Románica, en Filología Hispánica y Doctora en Filología Románica (Francés) por la Universidad de Valladolid. Profesora del Dpto. de Filología Francesa y Alemana en la Facultad de Ciencias Sociales, Jurídicas y de la Comunicación de la Universidad de Valladolid (Campus de Segovia). Su experiencia docente y de investigación se orientan al ámbito turístico; su labor investigadora se dirige tanto a aspectos pedagógicos de la enseñanza de la lengua francesa como a aspectos lingüísticos/discursivos/estilísticos de la lengua del francés del turismo, destacando el análisis realizado en la Tesis Doctoral sobre en "Estudio estilístico aplicado a un texto no literario: el folleto turístico" y numerosas ponencias/publicaciones

\section{María del Carmen Garrido Hornos}

Es Doctora en Filología Inglesa, miembro docente del Departamento de Filología Inglesa de la Universidad de Valladolid y Vicedecana de Relaciones Internacionales de la Facultad de Ciencias Sociales, Jurídicas y de la Comunicación en el Campus de Segovia. Su línea de investigación se centra básicamente en estudios de adaptación fílmica y análisis del discurso cinematográfico. Especialmente relevante ha sido su análisis de la adaptación filmográfica de la etapa americana de Alfred Hitchcock, cineasta sobre el que versó su Tesis Doctoral. Recientemente ha trabajado también sobre las nuevas tendencias de enseñanza universitaria, la repercusión del EEES en el aprendizaje del inglés como lengua extranjera y los nuevos modelos de tutoría universitaria 